\title{
ANÁLISES DA DINÂMICA ORÇAMENTÁRIA DOS MUNICÍPIOS BRASILEIROS: UMA APLICAÇÃO DA METODOLOGIA VAR COM DADOS EMPILHADOS
}

\author{
Bernardo P. Schettini *
}

\begin{abstract}
Resumo
O trabalho estima um VAR-painel usando dados de despesas totais, receitas tributárias e transferências correntes de 5.544 municípios brasileiros observados de 2002 a 2010. Uma sequência de hipóteses foi investigada por meio das estatísticas do teste de sobreidentificação. Entre os resultados encontrados, destacamos existirem evidências de mudança no comportamento fiscal dos municípios, mas que em média se mostrou compatível com a hipótese tax and spend e com o efeito flypaper das transferências sobre os gastos. Existe, aparentemente, muito mais dinâmica nas despesas do que na arrecadação própria. Tais resultados lançam luz sobre aspectos importantes da dinâmica orçamentária municipal.
\end{abstract}

Palavras-chave: Dinâmica orçamentária; Modelo VAR; Painel de dados; Municípios brasileiros.

\begin{abstract}
The paper estimates a panel-VAR using data on total expenditures, tax receipts and overall grants received for 5.544 Brazilian municipalities observed from 2002 through 2010. A sequence of hypotheses was investigated by the means of the overidentification test statistic. Among our main findings, we underscore there exists evidence of change in the fiscal behavior of the municipalities, but which in average support the tax and spend hypothesis, as well as the flypaper effect of grants over expenditures. We also find there is a lot more dynamics in expenditures compared to own revenues. Those results shed light on some important aspects of the municipal budget dynamics.
\end{abstract}

Keywords: Budget dynamics; VAR model; Panel data; Brazilian municipalities.

JEL classification: $\mathrm{C} 12, \mathrm{C} 33, \mathrm{H} 72$

\footnotetext{
* Instituto de Pesquisa Econômica Aplicada. E-mail: bernardo.schettini@ipea.gov.br
} 


\section{Introdução}

Este trabalho visa contribuir para o entendimento do comportamento fiscal dos municípios brasileiros, partindo de dados empilhados de 5.544 prefeituras no período de 2002 a 2010. A estratégia empírica envolveu a metodologia VAR-painel, objetivando contornar potenciais problemas relacionados à simultaneidade entre as variáveis fiscais, devido à indeterminação teórica das relações causais, e ao mesmo tempo investigar a dinâmica dos dados orçamentários. Estes são referentes à despesa total e receita tributária, além das transferências correntes.

A metodologia VAR com dados empilhados foi desenvolvida em HoltzEakin et al. (1988) e permite lidar com formas bastante gerais de dinâmica nos dados. Entre as aplicações do VAR-painel estão os estudos que investigam a dinâmica orçamentária municipal (Holtz-Eakin et al. 1989, Dahlberg \& Lindstrom 1998, Santolin et al. 2009). A ênfase reside na aplicação de uma sequência de testes de hipóteses, que procuram examinar a estabilidade no comportamento fiscal das prefeituras diante de choques, o tamanho da dinâmica nos dados, a influência das transferências sobre receitas tributárias e despesas agregadas, bem como confrontar certas teorias fiscais.

Existe uma relativa escassez de trabalhos na área de finanças públicas municipais no Brasil, não obstante os microdados sejam disponibilizados pelo Tesouro na base Finanças do Brasil - FINBRA. Os dados patrimoniais só passaram a serem divulgados de 1998 em diante, mas as informações sobre execução orçamentária utilizadas neste trabalho estão disponíveis a partir de 1989. No entanto, a Lei de Responsabilidade Fiscal (LRF), oficialmente Lei Complementar $\mathrm{n}^{\circ} 101$, de 04 de maio de 2000, promoveu uma melhora na qualidade desses dados, com notável crescimento do grau de cobertura.

Mas a punição prevista pela LRF não constitui incentivo suficiente para que os grandes recebedores de transferências não voluntárias prestem contas à Secretaria do Tesouro (STN). Para completar os buracos do FINBRA, partimos dos dados dos Relatórios Resumidos de Execução Orçamentária (RREO's) e de um procedimento de interpolação, implementado via técnicas de agrupamentos. Seguimos, dessa forma, a contribuição de Orair et al. (2011) no que se refere à imputação de dados anuais de finanças públicas dos municípios. $\mathrm{Na}$ sequência sumariam-se os principais resultados.

Os resultados encontrados evidenciam que as despesas dos governos municipais acomodam-se ao tamanho da carga tributária local, mostrando também que as transferências exercem influência própria sobre as despesas. Por seu turno, as receitas tributárias são exógenas, não reagindo a choques nos gastos. Pode-se dizer, portanto, que a hipótese tax and spend de Friedman se adéqua bem aos dados dos municípios brasileiros, bem como que as transferências exercem o efeito flypaper sobre os gastos. Diante de desequilíbrios no orçamento, o ajuste se dá por meio de adequação das despesas, em vez da arrecadação própria. Infere-se que mais receitas, na forma de arrecadação própria ou transferências intergovernamentais, resultam em novos gastos na esfera municipal.

O artigo está organizado em 4 seções, além desta breve introdução. A seção 2 mostra a estratégia econométrica, além de aplicações com base em dados municipais de finanças públicas. A seção 3 descreve os dados, enquanto a seção 4 mostra os principais resultados e análises. A seção 5 sintetiza e conclui. 


\section{Metodologia VAR-painel e análises da política fiscal}

Esta seção mostra o modelo empírico, além de um detalhamento sobre a estimação e testes de hipóteses, bem como faz um levantamento da literatura aplicada nacional e internacional sobre a temática da dinâmica na execução orçamentária municipal.

\subsection{Modelo de painel dinâmico}

A análise aplicada realizada ao longo deste trabalho combina a lógica do modelo VAR com a ideia do painel. Ao trabalhar com a estrutura VAR, busca-se contornar potenciais problemas de simultaneidade, decorrentes da indeterminação teórica nas relações causais. A estrutura de painel, por seu turno, permite controlar para a heterogeneidade individual não observada entre as unidades de tomada de decisão e contornar problemas relacionados à agregação das preferências (Dahlberg \& Lindstrom 1998).

O modelo de regressão abaixo parte da ideia de que a evolução das despesas $(x)$ é explicada por suas próprias defasagens e pelos valores passados das receitas tributárias $(r)$ e de transferências $(g)$ :

$$
\begin{gathered}
x_{i t}=\alpha_{0}+\sum_{l=1}^{m} \alpha_{l} x_{i, t-l}+\sum_{l=1}^{m} \delta_{l} r_{i, t-l}+\sum_{l=1}^{m} \pi_{l} g_{i, t-l}+f_{i}+u_{i t}, \\
(i=1, \ldots, N ; t=m+1, \ldots, T) .
\end{gathered}
$$

A exposição que segue foca na equação das despesas, mas existe o simétrico da equação (1) para as receitas tributárias e de transferências.

$\mathrm{Na}$ prática, o efeito fixo $f_{i}$, possivelmente correlacionado com os regressores, é removido da equação ao se trabalhar com a primeira diferença das variáveis:

$$
\begin{gathered}
\Delta x_{i t}=\sum_{l=1}^{m} \alpha_{l} \Delta x_{i, t-l}+\sum_{l=1}^{m} \delta_{l} \Delta r_{i, t-l}+\sum_{l=1}^{m} \pi_{l} \Delta g_{i, t-l}+\Delta u_{i t}, \\
(i=1, \ldots, N ; t=m+2, \ldots, T) .
\end{gathered}
$$

A estimação é, em geral, feita por GMM em diferença (Holtz-Eakin et al. 1988, Arellano \& Bond 1991) ou sistema GMM (Arellano \& Bover 1995, Blundell \& Bond 1998). Mas não existe ponto passivo na discussão sobre quais (e a quantidade de) condições de momento utilizar. Ver, por exemplo, Roodman $(2006)^{1}$.

A equação (1) pode ser generalizada para permitir que os coeficientes variem ao longo do tempo:

$$
\begin{gathered}
x_{i t}=\alpha_{0 t}+\sum_{l=1}^{m} \alpha_{l t} x_{i, t-l}+\sum_{l=1}^{m} \delta_{l t} r_{i, t-l}+\sum_{l=1}^{m} \pi_{l t} g_{i, t-l}+\psi_{t} f_{i}+u_{i t}, \\
(i=1, \ldots, N ; t=m+1, \ldots, T) .
\end{gathered}
$$

\footnotetext{
${ }^{1}$ É verdade que, do ponto de vista da eficiência, todas as condições disponíveis deveriam ser exploradas. Mas a proliferação de instrumentos, muitos deles possivelmente fracos, pode resultar em viés devido à sobreparametrização das variáveis endógenas.
} 
É possível elencar alguns fatores que podem levar a mudança paramétrica. É amplamente reconhecido, para citar os exemplos mais evidentes, que mudanças na legislação, o ciclo político e as próprias eleições, além de choques macroeconômicos, impactam o comportamento fiscal das prefeituras. Certamente, tais fenômenos aumentam a incerteza com relação à adequação do modelo linear.

A não estacionariedade dos coeficientes no painel é uma área pouco explorada em trabalhos empíricos. Mas, na presença de quebra estrutural, algumas condições de momento podem não ser válidas e isso pode levar a inconsistência (De Wachter \& Tzavalis 2004). Os desenvolvimentos recentes na área evidenciam a importância prática de se trabalhar com a hipótese de quebra (Andrews \& Lu 2001, De Wachter \& Tzavalis 2005) 2 .

Tendo dito isso, julga-se não ser prudente descartar de saída a possibilidade de inovações afetarem a política orçamentária na esfera municipal. A equação (3) permite que tais choques afetem tanto o nível da função como os coeficientes de inclinação. É importante ter claro que nessa especificação o termo idiossincrático também pode ser deslocado, porém tal mudança se dá em proporção igual entre os municípios.

Na prática, para eliminar este efeito individual não observável que varia ao longo do tempo, subtrai-se de (3) a mesma equação em $t-1$ multiplicada por $\lambda_{t}=\psi_{t} / \psi_{t-1}$. Ver também Chamberlain (1992) e Wooldridge (1991). Com esse procedimento de "quase-diferenciação", reordenando-se chega-se a:

$$
\begin{gathered}
x_{i t}=a_{t}+\sum_{l=1}^{m+1} c_{l t} x_{i, t-l}+\sum_{l=1}^{m+1} d_{l t} r_{i, t-l}+\sum_{l=1}^{m+1} p_{l t} g_{i, t-l}+v_{i t}, \\
(i=1, \ldots, N ; t=m+2, \ldots, T) .
\end{gathered}
$$

onde:

$$
\begin{aligned}
& a_{t}=\alpha_{0 t}-\lambda_{t} \alpha_{0, t-1} ; v_{i t}=u_{i t}-\lambda_{t} u_{i, t-1} ; \\
& c_{1 t}=\lambda_{t}+\alpha_{1 t} ; d_{1 t}=\delta_{1 t} ; p_{1 t}=\pi_{1 t} ; \\
& c_{m+1, t}=-\lambda_{t} \alpha_{m, t-1} ; d_{m+1, t}=-\lambda_{t} \delta_{m, t-1} ; p_{m+1, t}=-\lambda_{t} \pi_{m, t-1} ; \\
& c_{l t}=\alpha_{l t}-\lambda_{t} \alpha_{l-1, t-1} ; d_{l t}=\delta_{l t}-\lambda_{t} \delta_{l-1, t-1} ; p_{l t}=\pi_{l t}-\lambda_{t} \pi_{l-1, t-1} ; \\
& \quad(l=2, \ldots, m) .
\end{aligned}
$$

A equação (4) constitui o modelo de partida, com o qual se trabalha inicialmente. Uma sequência de testes de hipóteses é então aplicada nas equações de despesas e receitas tributárias para checar o tamanho e a natureza da dinâmica orçamentária, e finalmente selecionar as especificações que aparentam ser mais consistentes com os dados.

\subsection{Estimação e testes de hipóteses}

No que segue, mostramos os passos da estimação GMM e os principais pontos relacionados aos testes de hipóteses. A exposição foi baseada em Holtz-Eakin et al. (1988), que pode ser consultado para se checar os detalhes.

\footnotetext{
${ }^{2}$ Há obviamente um custo para se aumentar o modelo e permitir quebras, na forma de perda de graus de liberdade na estimação. Note-se que no caso concreto o número de parâmetros a ser estimado pode se tornar dramaticamente grande dependendo do tamanho da dinâmica que se pretende permitir nos dados.
} 
A estimação é feita com base nas seguintes condições de ortogonalidade:

$$
E\left[x_{i s} v_{i t}\right]=E\left[r_{i s} v_{i t}\right]=E\left[g_{i s} v_{i t}\right]=0 \quad(s<(t-1)),
$$

e o vetor de variáveis instrumentais disponíveis para se estimar as equações (2) e (4) é o seguinte:

$$
Z_{i t}=\left[1, x_{i, t-2}, \ldots, x_{i 1}, r_{i, t-2}, \ldots, r_{i 1}, g_{i, t-2}, \ldots, g_{i 1}\right] .
$$

Para atingir identificação, é preciso que o número de instrumentos seja ao menos o mesmo do que o de variáveis do lado direito do modelo de regressão. A dimensão de $Z_{i t}$ é $3 t-5$. Há $3 m+1$ regressores na equação (2), sendo que a estimação pode ser feita para $t \geq m+2$. Mas temos $3 m+4$ variáveis do lado direito da equação (4), e a condição de ordem exige $t \geq m+3^{3}$.

Lançando mão da notação de álgebra matricial, facilita-se a exposição que segue. Sejam $X_{t}=\left[x_{1 t}, \ldots, x_{N t}\right]^{\prime}, R_{t}=\left[r_{1 t}, \ldots, r_{N t}\right]^{\prime}$ e $G_{t}=\left[g_{1 t}, \ldots, g_{N t}\right]^{\prime}$ os vetores $N \times 1$ de dados e $W_{t}=\left[\iota, X_{t-1}, \ldots, X_{t-m-1}, R_{t-1}, \ldots, R_{t-m-1}, G_{t-1}\right.$,

$\left.\ldots, G_{t-m-1}\right]$ a matriz de variáveis explicativas $N \times(3 m+4)$, onde $\iota$ é um vetor $N \times 1$ de 1 's. O vetor de parâmetros $B_{t}=\left[a_{t}, c_{1 t}, \ldots, c_{m+1, t}, d_{1 t}, \ldots, d_{m+1, t}\right.$,

$\left.p_{1 t}, \ldots, p_{m+1, t}\right]^{\prime}$ tem dimensão $(3 m+4) \times 1$.

A equação (4) pode ser representada por:

$$
X_{t}=W_{t} B_{t}+V_{t} \quad(t=(m+3), \ldots, T),
$$

onde $V_{t}=\left[v_{1 t}, \ldots, v_{N t}\right]^{\prime}$ é o vetor $N \times 1$ de erros de previsão transformados. $\mathrm{O}$ próximo passo consiste em empilhar essas $T-m-2$ equações:

$$
X=W B+V .
$$

onde os vetores $X=\left[X_{m+3}^{\prime}, \ldots, X_{T}^{\prime}\right]^{\prime}$ e $V=\left[V_{m+3}^{\prime}, \ldots, V_{T}^{\prime}\right]^{\prime}$ são $(T-m-2) N \times 1$; o vetor $B=\left[B_{m+3}^{\prime}, \ldots, B_{T}^{\prime}\right]^{\prime}$ é $(T-m-2)(3 m+4) \times 1$; e a matriz $W$, de dimensão $(T-m-2) N \times(T-m-2)(3 m+4)$, é diagonal por blocos com as entradas na diagonal, i.e. $W=\operatorname{diag}\left[W_{m+3}^{\prime}, \ldots, W_{T}^{\prime}\right]$.

A matriz $Z_{t}=\left[\iota, X_{t-2}, \ldots, X_{1}, R_{t-2}, \ldots, R_{1}, G_{t-2}, \ldots, G_{1}\right]$ contém as $(3 t-5)$ variáveis que, em principio, se qualificam como instrumentos válidos para a regressão do período $t$. As condições de momento crescem rapidamente em $t$, o que é evidenciado pela dimensão de $Z=\operatorname{diag}\left[Z_{m+3}, \ldots, Z_{T}\right]$, de tamanho $(T-m-2) N \times \sum_{t=m+3}^{T}(3 t-5)$. A estimação é feita via 3SLS, sendo numericamente igual ao estimador GMM em diferença.

No primeiro estágio, aplica-se 2SLS em cada $t \geq m+3$ para se estimar o vetor:

$$
\tilde{B}_{t}=\left[W_{t}^{\prime} Z_{t}\left(Z_{t}^{\prime} Z_{t}\right)^{-1} Z_{t}^{\prime} W_{t}\right]^{-1} W_{t}^{\prime} Z_{t}\left(Z_{t}^{\prime} Z_{t}\right)^{-1} Z_{t}^{\prime} X_{t},
$$

e então obter os resíduos $\tilde{V}_{t}=X_{t}-W_{t} \tilde{B}_{t}$ usando essas estimativas preliminares.

No segundo estágio, um estimador consistente da matriz de covariância $\Omega=E\left\{Z^{\prime} V V^{\prime} Z\right\}$ é formado usando $\tilde{V}_{t}$ e $Z_{t}$ :

\footnotetext{
${ }^{3}$ Para identificar os parâmetros da equação não transformada (3), é preciso ter $2 T \geq 5 m+4$. Isso porque é possível estimar os parâmetros da equação (4) para $T-m-2$ períodos e, sem contar as constantes, há um total de $(T-m-2) 3(m+1)$ parâmetros na equação transformada, o que significa um total de $(T-m-2)+(T-m-1) 3 m$ parâmetros na equação (3). Ocorre que o objetivo não é identificar tais parâmetros, dado que é possível implementar os testes de hipóteses como base na equação transformada.
} 


$$
\tilde{\Omega}=\sum_{i=1}^{N}\left(v_{i r} v_{i s} Z_{i r}^{\prime} Z_{i s}\right),
$$

em que (para $t=r, s) v_{i t}$ é o $i$-ésimo elemento de $\tilde{V}_{t}$ e $Z_{i t}$ é a $i$-ésima linha de $Z_{t}$.

O terceiro estágio consiste na estimação GLS, usando a matriz de covariância estimada e todas as observações disponíveis, do vetor de parâmetros como um todo:

$$
\hat{B}=\left[W_{t}^{\prime} Z_{t}(\tilde{\Omega})^{-1} Z_{t}^{\prime} W_{t}\right]^{-1} W_{t}^{\prime} Z_{t}(\tilde{\Omega})^{-1} Z_{t}^{\prime} X_{t}
$$

Para testar hipóteses de restrições lineares, comparam-se a soma dos quadrados dos resíduos (SSR) dos modelos restrito e irrestrito. Mais concretamente, considere a hipótese nula:

$$
H_{0}: B=H \gamma+G
$$

onde $\gamma$ é o vetor $k \times 1$ de parâmetros restritos, $H$ é uma matriz constante $(T-m-2)(3 m+4) \times k$ e $G$ tem a mesma dimensão de $B$. O modelo restrito é dado por:

$$
\tilde{X}=X-W G=W H \gamma+V=\tilde{W} \gamma+V .
$$

As relações abaixo definem $Q$ e $Q_{R}$ como a $S S R$ dos modelos irrestrito e restrito, respectivamente:

$$
\begin{aligned}
Q & =(X-W \hat{B})^{\prime} Z_{t}(\tilde{\Omega})^{-1} Z_{t}^{\prime}(X-W \hat{B}) / N, \\
Q_{R} & =(X-W \hat{\gamma})^{\prime} Z_{t}(\tilde{\Omega})^{-1} Z_{t}^{\prime}(X-W \hat{\gamma}) / N .
\end{aligned}
$$

A estatística $Q$ segue uma distribuição qui-quadrada à medida que $N$ cresce, com graus de liberdade igual à diferença entre a quantidade de instrumentos (número de linhas em $Z^{\prime} V$ ) e o número de parâmetros (dimensão de $B)$, podendo ser aplicada para um teste conjunto da especificação inicial do modelo e das condições de momento (teste de sobreidentificação).

Restrição lineares podem ser testadas com o auxílio da estatística $L$, que tem a forma do numerador da estatística $F:^{4}$.

$$
L=Q_{R}-Q
$$

A estatística $L$ é distribuída conforme uma qui-quadrada com graus de liberdade dados pela diferença entre o número de graus de liberdade de $Q_{R} \mathrm{e}$ $Q$. É importante notar que a mesma matriz de covariância deve ser utilizada ao se computar a $S S R$ dos modelos restrito e irrestrito.

\footnotetext{
${ }^{4}$ Holtz-Eakin et al. (1988) enfatizam que as estatísticas são assintoticamente independentes em uma sequência de testes quando a hipótese de não causalidade está aninhada com as hipóteses de estacionariedade dos parâmetros e do número de defasagens.
} 


\subsection{Aplicações}

A aplicação do modelo VAR-painel aos dados fiscais municipais é motivada pela indeterminação teórica no que se refere às relações causais entre as variáveis de receita e despesa.

Conforme notaram Santolin et al. (2009), análises da política fiscal municipal brasileira muitas vezes não se atentam para a possibilidade de viés de simultaneidade. Os autores trabalharam com dados das despesas dos municípios de Minas Gerais com pessoal e de investimento entre 1995 e 2005, procurando avaliar os impactos da LRF sobre estes grupos de natureza de despesa.

As análises da política fiscal municipal brasileira são, de uma maneira geral, voltadas para avaliar os impactos da LRF (Menezes 2005, Chieza et al. 2009, Menezes \& Toneto Jr. 2006) ou mensurar a influência das transferências sobre o esforço de arrecadação tributária e o descontrole dos gastos (Postali \& Rocha 2009, Orair \& Alencar 2010, Queiroz \& Postali 2010, Mattos et al. 2011). Destaca-se que a influência das transferências sobre a execução orçamentária é um tema central para o aprimoramento do pacto federativo brasileiro.

No VAR-painel, a influência das transferências sobre a arrecadação tributária e as despesas totais pode ser avaliada por intermédio de testes de exclusão de Granger. Interessa saber se valores passados das transferências melhoram a previsão das receitas tributárias ou dos gastos totais. Para confrontar tais hipóteses com os dados, faz-se necessário aplicar testes de restrição ${ }^{5}$.

A literatura aplicada internacional é voltada para testar quatro hipóteses, lançadas pelos clássicos da literatura econômica na área de finanças públicas e política fiscal.

[1] Para Friedman (1978), os gastos públicos se acomodam, para cima ou para baixo, ao tamanho da carga tributária. A mesma relação causal resulta da teoria de que os burocratas procuram maximizar o orçamento e não expandem os gastos devido a limites de endividamento (Niskanen 1971). Assim, tem-se a hipótese de tax and spend.

[2] Na visão de Peacock \& Wiseman (1979), as despesas públicas crescem em épocas de crises econômicas, sendo o equilíbrio fiscal atingido posteriormente através do aumento na arrecadação. Para Barro (1979), os gastos públicos são exógenos e determinam a arrecadação, que é distribuída ao longo do tempo (tax smoothing). A hipótese de spend and tax representa esta visão.

[3] Uma relação de simultaneidade poderia ocorrer se os (representantes dos) cidadãos de cada município definissem, para cada ano, um esquema ótimo de tributação e gastos baseando-se nos custos e benefícios marginais, em conformidade com a teoria do eleitor mediano (Black 1948).

[4] Caso receitas e gastos fossem definidos a partir de "regras de bolso", e.g. como fatias constantes do PIB municipal, ter-se-ia uma relação de independência (Hoover \& Sheffrin 1992).

Holtz-Eakin et al. (1989) analisaram os dados fiscais de 171 municípios americanos no período entre 1972 e 1980, concluindo que a relação intertemporal entre receitas e gastos pode ser resumida em um ou dois anos. Os valores passados das receitas ajudaram a prever os gastos correntes, mas as despesas

\footnotetext{
${ }^{5} \mathrm{~A}$ aplicação dos testes de exclusão de Granger para inferir causalidade é bastante controversa, dado que se refere a um tipo bastante restrito de relação causal. Este trabalho não aprofunda nessa discussão. Cabe notar, no entanto, que a proposta do estudo diferencia-se das aplicações com base no painel convencional, em que a noção de causalidade é baseada na identificação do efeito parcial de uma variável exógena.
} 
não melhoraram a previsão das receitas. Foram encontradas evidências de instabilidade dos parâmetros na equação dos gastos.

Dahlberg \& Johansson (1998) trabalharam com dados de um painel de 265 municípios suecos no período de 1974 a 1987 e encontraram maior dinâmica, sendo que quatro anos de defasagens se mostraram necessários para descrever os dados. Os autores também encontraram evidência de instabilidade na equação de despesas. No que se refere à causalidade no sentido de Granger, os testes de exclusão indicaram que as despesas e as transferências melhoraram a previsão das receitas tributárias. Há também causalidade das transferências para as despesas, mas a receita tributária não ajudou na previsão dos gastos totais.

Dahlberg \& Johansson (2000) também analisaram um painel de $265 \mathrm{mu}$ nicípios suecos, mas o período amostral vai de 1979 a 1987 . Os testes de hipótese foram realizados a partir de valores críticos bootstrapping, em vez dos assintóticos. Um ano de defasagem pareceu suficiente para resumir a dinâmica na equação das despesas. Nenhuma defasagem sobreviveu aos testes de significância nas equações das receitas tributárias e transferências. Os testes de exclusão na equação de despesas, bem como a própria magnitude dos parâmetros, indicaram que as receitas próprias são um determinante mais importante dos gastos do que as transferências.

Moisio (2000) analisou o caso finlandês com dados para 436 municípios, comparando os resultados de dois períodos amostrais. De 1985 a 1992, a maior parte das transferências obedecia a um critério de matching grant e eram vinculadas a despesas específicas, enquanto de 1993 a 1999 não havia vinculação e as transferências eram baseadas em fórmulas. O autor incluiu, adicionalmente, uma equação para os empréstimos feitos pelos governos municipais, dado que era permitido o financiamento através de dívida.

Mesmo trabalhando com dois períodos amostrais, Moisio mostrou haver evidências de instabilidade paramétrica. No primeiro período, apenas os coeficientes da equação das despesas se mostraram estáveis. No segundo, apenas os coeficientes na equação dos empréstimos não variaram. De 1985 a 1992, as despesas municipais ajudaram a prever as receitas tributárias, mas não o contrário. De 1993 a 1999, há evidências de que as receitas tributárias ajudaram na previsão dos gastos, bem como as despesas melhoraram a previsão das receitas. No que se refere às transferências e ao endividamento, Moisio encontrou evidência de que essas variáveis causaram tanto as receitas tributárias como as despesas totais no sentido de Granger.

\section{Base de dados}

Os dados da execução orçamentária (receitas e despesas) dos municípios brasileiros estão disponíveis de 1989 em diante na base FINBRA, disponibilizada pela STN. Adicionalmente, o FINBRA passa a divulgar informações patrimoniais (ativos e passivos) em 1998. Atualmente, essa base de dados contém 
informações tanto da execução orçamentária quanto patrimoniais ${ }^{6,7}$.

Mas, ao lidar com a base FINBRA, é preciso contornar alguns problemas. Entre estes, destacam-se: i) mudanças na territorialidade brasileira com a criação de municípios; ii) mudanças conceituais na contabilidade pública brasileira (alterações no plano de contas); iii) crescente nível de detalhamento das informações contábeis; iv) erros na prestação de contas; e v) ausência de dados (missings).

Com relação à intensa criação de municípios durante a década de 1990, alguns autores optam por trabalhar com o conceito de Áreas Mínimas Comparáveis (AMC's) que consiste "de um painel de áreas geográficas que possibilita comparações intertemporais consistentes dos dados demográficos, econômicos e sociais disponíveis em nível municipal nos censos realizados de 1872 a 2000 " (Reis et al. 2007, p. 1). Mas é importante ter claro que, ao se trabalhar com as AMC's, perde-se a ideia de que as unidades cross-section do painel são unidades de tomada de decisão. Isso porque as AMC's não são regiões administrativas e cabe às prefeituras municipais arrecadar e alocar o orçamento. Este trabalho utiliza dados de 2002 a 2010, período em que houve poucas mudanças na territorialidade do país ${ }^{8}$.

Mudanças na definição das variáveis e alterações no nível de detalhamento dos dados significam não ser possível comparar determinados dados desagregados ao longo de vários anos. Mas este trabalho objetiva analisar os dados agregados, e esse detalhe em particular não representa uma limitação relevante. A principal dificuldade com relação aos dados é decorrente de erros na prestação de contas e da ausência de dados para alguns municípios em determinados anos. Os erros na prestação de contas estão ligados ao treinamento dos contadores municipais e, na prática, é virtualmente impossível contornálos. Mas note-se que a LRF promoveu uma melhora na qualidade dos dados, com notável crescimento da cobertura ${ }^{9}$. Ocorre que mesmo com a punição

\footnotetext{
${ }^{6}$ Segundo consta nas "notas explicativas" que acompanham as bases de dados do FINBRA, "sua elaboração decorre das disposições dos artigos 11 e 112 da Lei 4.320, de 17 de março de 1964, e do artigo 51 da Lei Complementar $n^{\circ} 101$, de 4 de maio de 2000, relacionadas com a obrigatoriedade da organização e publicação, até 30 de junho de cada ano, dos balanços consolidados das contas dos entes da Federação relativas ao ano anterior, o que vem sendo feito por intermédio da STN".

${ }^{7}$ Todo ano, pode existir mais de uma versão do FINBRA, conforme notaram Orair \& Alencar (2010). De acordo com a LRF, os municípios devem transmitir seus dados contábeis referentes ao exercício fiscal do ano anterior até o mês de abril, cabendo à STN divulgar a Consolidação das Contas Públicas até o mês de junho. A primeira versão do FINBRA contém apenas os municípios que encaminharam seus dados na primeira metade do ano, a tempo de serem compilados pela STN. Ao longo do ano, a STN recebe e processa mais dados, daí que a versão completa do FINBRA é geralmente divulgada em agosto ou setembro. Mesmo essa versão, que possui uma cobertura maior de municípios, está sujeita a revisões pela STN nos anos subseqüentes.

${ }^{8}$ A Emenda Constitucional no 15 , de 12 de setembro de 1996, alterou o $\S 4^{\circ}$ do art. 18 da Constituição Federal, que versa sobre a "criação, incorporação, fusão e desmembramento de Municípios". As mudanças na territorialidade "far-se-ão por lei estadual, dentro do período determinado por Lei Complementar Federal, e dependerão de consulta prévia, mediante plebiscito, às populações dos Municípios envolvidos, após a divulgação de Estudos de Viabilidade Municipal, apresentados e publicados na forma da lei".

${ }^{9} \mathrm{O} \S 1^{\circ}$ do art. 51 da LRF estabelece os prazos para os estados e municípios encaminharem suas contas para que o Poder Executivo da União promova, até 30 de junho, a consolidação das contas dos entes federados relativas ao exercício anterior. O prazo para os municípios é 30 de abril e o dos estados 30 de maio. $\mathrm{O} \S 2^{\circ}$ pune os entes que não transmitirem as informações ou descumprirem os prazos. "O descumprimento dos prazos previstos neste artigo impedirá, até que a situação seja regularizada, que o ente da Federação receba transferências voluntárias e contrate operações de crédito, exceto as destinadas ao refinanciamento do principal atualizado da dívida
} 
prevista pela LRF, vários municípios não transmitem as informações à STN, ou o fazem com grande atraso.

Para imputar os dados ausentes na matriz de dados do FINBRA procedeuse da seguinte maneira. No caso dos municípios fluminenses que não prestaram contas à STN com regularidade, as informações contábeis foram obtidas junto ao Tribunal de Constas do Estado do Rio de Janeiro (TCE-RJ) ${ }^{10}$. É inviável replicar tal procedimento para todos os estados da federação, tendo sido adotado um método de interpolação. Faz-se uso de técnicas de agrupamento (clusters), objetivando formar grupos homogêneos com base na posição geográfica, população e PIB por habitante (Orair et al. 2011) ${ }^{11}$. Uma descrição sumária do método é feita na sequência.

O método consiste de uma técnica hierárquica aglomerativa, baseada em medida de similaridade. Optou-se por trabalhar com a distância de Mahalanobis ${ }^{12}$. A principal vantagem reside na ponderação pela matriz de variânciacovariância. Deste modo, impede-se que uma medida com variabilidade bem maior que as demais afete o agrupamento de forma desproporcional, bem como consideram-se possíveis relações lineares entre variáveis, evitando problemas de multicolinearidade. Para se chegar à partição final, adotou-se o método de Ward, para minimizar o desvio-padrão entre os dados de cada grupo.

Tal análise foi feita desconsiderando os 300 municípios com maiores PIB por habitante. O procedimento resultou em uma partição formada de 13 grupos, tendo a escolha se baseado nas medidas de Pseudo $F$ e Pseudo $T$, além da evolução do $\mathrm{R}^{2}$ durante o processo. Em análises dos empates entre as distâncias, por meio de 15 permutações aleatórias diferentes, os grupos se mostraram estáveis, não havendo influência de empates na partição final. Por fim, a imputação foi feita aplicando-se os índices de evolução de cada grupo nos municípios com informações ausentes.

Nas análises empíricas, parte-se de dados anuais de execução orçamentária de 5.544 municípios brasileiros observados de 2002 a $2010(N=5.544, T=$ $9)^{13}$. No que se refere ao impacto da interpolação, destaca-se que a aplicação da técnica abrangeu cerca de $4,6 \%$ do painel de dados. Isso porque, de um total de 49.896 observações, foram imputados 2.314 dados de despesas totais, 2.324 de receitas tributárias e 2.303 de transferências correntes.

Tendo em vista os objetivos desta pesquisa, trabalhamos com as variáveis em nível agregado no VAR-painel. Analisamos o comportamento conjunto da "despesa total" $(X)$, "receitas tributárias" $(R)$, e "transferências correntes"

\footnotetext{
mobiliária".

${ }^{10}$ Os RREO's dos municípios fluminenses são publicados em http://www.tce.rj.gov . $\mathrm{br} /$.

${ }^{11}$ As variáveis de posição geográfica foram obtidas a partir da Malha Municipal Digital do Brasil de 2005, um produto cartográfico do Instituto Brasileiro de Geografia e Estatística (IBGE). O PIB per capita e a população são estimativas também do IBGE.

${ }^{12}$ Considerando duas observações $i$ e $j$ de um vetor aleatório $X$ com matriz de variânciacovariância $\sum$, a distância $d_{i j}^{M B}$ entre dois vetores $X_{i}$ e $X_{j}$ é dada por: $d_{i j}^{M B}=\left(X_{i}-X_{j}\right)^{\prime} \Sigma^{-1}\left(X_{i}-\right.$ $\left.X_{j}\right)$.

${ }^{13}$ Além de Brasília - DF e Fernando de Noronha - PE que não fazem parte do FINBRA, 19 municípios foram excluídos do painel. Há alguns casos de municípios criados durante o período amostral (Figueirão - MS, Ipiranga do Norte - MT, Itanhangá - MT, Aroeiras do Itatim - PI, Nazária - PI), além de prefeituras que não prestaram contas em nenhum ano (Gongogi - BA, São Vicente Ferrer - MA, Cachoeira do Piriá - PA), ou que reportaram receitas tributárias (Calçoene - AP, Mazagão - AP, Goianira - GO, Altamira do Maranhão - MA, Apicum-Açu - MA, Brejo de Areia - MA, Cândido Mendes - MA, Concórdia do Pará - PA, Maracanã - PA e São Luiz - RR) e de transferências (Franco da Rocha - SP) nulas em algum ano.
} 
Tabela 1: Plano de contas - receitas e despesas por categorias e grupos, válido de 2002 em diante

\begin{tabular}{rlrc}
\hline CÓD & CONTAS DE RECEITAS & CÓD & CONTAS DE DESPESAS \\
\hline 1 & RECEITA TOTAL & 91 & DESPESA TOTAL \\
2 & RECEITAS CORRENTES & 92 & DESPESAS CORRENTES \\
3 & Receita Tributária & 93 & Pessoal e Encargos Sociais \\
13 & Receitas de Contribuições & 113 & Juros e Encargos da Dívida \\
16 & Receita Patrimonial & 114 & Outras Despesas Correntes \\
21 & Receita Agropecuária & 158 & DESPESAS DE CAPITAL \\
22 & Receita Industrial & 159 & Investimentos \\
23 & Receita de Serviços & 160 & Inversões Financeiras \\
24 & Transferências Correntes & 161 & Amortização da dívida \\
58 & Outras Receitas Correntes & 162 & SUPERÁVIT/DÉFICIT=(1-91) \\
63 & RECEITAS DE CAPITAL & & \\
64 & Operações de Crédito & & \\
67 & Alienação de Bens & & \\
70 & Amortização de Empréstimos & \\
71 & Transferências de Capital & & \\
81 & Transferência de Convênios da & & \\
& União e de suas Entidades & & \\
82 & Transferência de Convênios dos & & \\
83 & Estados & & \\
84 & Transferência de Convênios dos & & \\
86 & Municípios e de suas Entidades & & \\
& Transferência de Convênios de & & \\
& Instituições Privadas & Outras Receitas de Capital & \\
\hline
\end{tabular}

Fonte: FINBRA/STN.

$(G)$. A Tabela 1 mostra um resumo do plano de contas atualmente aplicado na contabilidade pública brasileira, o que permite se ter uma ideia do nível de agregação desses dados ${ }^{14}$.

As receitas tributárias incluem os impostos, as taxas e contribuições de melhoria ${ }^{15}$. As transferências intergovernamentais perfazem, em grande parte dos casos, quase que a totalidade das transferências correntes, que incluem ainda as transferências de instituições privadas, do exterior, de pessoas e de convênios. As despesas totais são formadas pelos gastos correntes e de capi$\operatorname{tal}^{16}$.

A Tabela 2 descreve os dados. As estatísticas "entre" (between) retratam os dados partindo da média de cada município ao longo do tempo. As esta-

\footnotetext{
${ }^{14}$ Note-se que esse plano de contas se refere ao período de 2002 em diante, quando houve uma mudança na forma de divulgação dos dados.

${ }^{15}$ Os impostos municipais são o "Imposto Predial e Territorial Urbano" (IPTU), o "Imposto sobre Serviços de Qualquer Natureza" (ISSQN) e o "Imposto sobre a Transmissão de Bens Imóveis" (ITBI), além do "Imposto de Renda Retido na Fonte" (IRRF) referente aos funcionários públicos locais. As taxas recolhidas pelas prefeituras são referentes ao "Exercício do Poder de Polícia" e "Prestação de Serviços".

${ }^{16}$ Entre os gastos correntes, há três grupos de natureza de despesa. O grupo "pessoal e encargos sociais" é composto das despesas orçamentárias com pessoal ativo, inativo e pensionistas. Os "juros e encargos da dívida" constituem o componente financeiro dos gastos correntes, englobando o pagamento de juros, comissões e outros encargos de operações de crédito internas e externas contratadas, além da dívida mobiliária. As "outras despesas correntes" se referem à aquisição de material de consumo, pagamento de diárias, contribuições, subvenções, auxílioalimentação, auxílio-transporte, além de outras despesas correntes que não se enquadram nos demais grupos. Trata-se, basicamente, do consumo intermediário dos municípios.
} 
tísticas "dentro" (within) se referem aos desvios com relação à média de cada município.

Todas as variáveis foram mensuradas em termos reais (preços médios de 2010, em R\$) e por habitante. O deflator adotado foi o Índice de Preços ao Consumidor Amplo (IPCA) do IBGE. Os dados da população municipal são as estimativas anuais também do IBGE.

Tabela 2: Estatística descritiva - receitas e despesas municipais por habitante, 2002 a 2010

\begin{tabular}{llccrcc}
\hline Variável & & Média & Desvio-padrão & \multicolumn{1}{c}{ Min } & \multicolumn{1}{c}{ Max } & Observações \\
\hline$X$ & Total & $1.408,05$ & 920,82 & 161,70 & $104.322,40$ & $N \times T=49.896$ \\
& Entre & & 722,04 & 415,62 & $12.347,62$ & $N=5.544$ \\
& Dentro & & 571,53 & $-10.295,49$ & $93.382,81$ & $T=9$ \\
\multirow{4}{*}{$R$} & & & & & & \\
& Total & 89,51 & 129,10 & 0,77 & $3.740,03$ & $N \times T=49.896$ \\
& Entre & & 115,98 & 6,01 & $1.713,78$ & $N=5.544$ \\
& Dentro & & 56,72 & $-950,15$ & $3.105,39$ & $T=9$ \\
$G$ & & & & & & \\
& Total & $1.322,41$ & 904,38 & 80,67 & $107.239,40$ & $N \times T=49.896$ \\
& Entre & & 703,38 & 321,92 & $12.660,56$ & $N=5.544$ \\
& Dentro & & 568,54 & $-10.733,11$ & $95.901,30$ & $T=9$ \\
\hline
\end{tabular}

Fonte: FINBRA e RREO's.

Elaboração própria.

As variáveis foram descompostas em "entre" $\left(\bar{x}_{i .}\right)$ e "dentro" $\left(x_{i t}-\bar{x}_{i .}+\bar{x}_{. .}\right)$, onde

$\bar{x}_{i .}=(1 / T) \sum_{t=1}^{T} x_{i t}$ é média da variável durante o período amostral para o município $i \mathrm{e}$

$\bar{x}_{. .}=(1 / T) \sum_{t=1}^{N} \bar{x}_{i}$. é a média global.

No que se refere à arrecadação, nota-se que a média por habitante da receita tributária é cerca de quinze vezes menor do que a de transferências nos municípios brasileiros. A despesa total média por habitante é ainda superior.

Um ponto importante diz respeito à variância dos dados. Observa-se que a maior parte da variabilidade se dá entre os municípios em determinado ano, e não ao longo dos anos para uma dada prefeitura. Apesar do painel levar em conta tanto a informação cross-section quanto a temporal, esse padrão de dispersão significa que as estimativas dos modelos de regressão devem refletir principalmente a variabilidade que existe entre as unidades longitudinais.

A Figura 1 mostra a trajetória das receitas e despesas municipais agregadas, definidas como o somatório dos dados empilhados municipais em cada ponto no tempo. Evidencia-se o impacto de choques macroeconômicos sobre as receitas próprias e de transferências, bem como uma possível influência das eleições sobre as despesas agregadas.

\section{Testes de hipóteses no VAR-painel}

Esta seção examina empiricamente o seguinte conjunto de questões com base nos dados de execução orçamentária das prefeituras:

[1] Há evidências de não estacionariedade dos parâmetros nas equações de despesas orçamentárias e receitas tributárias? Pode-se descartar a hipótese de que o comportamento fiscal dos gestores municipais é afetado por choques que afetam todas as prefeituras? 


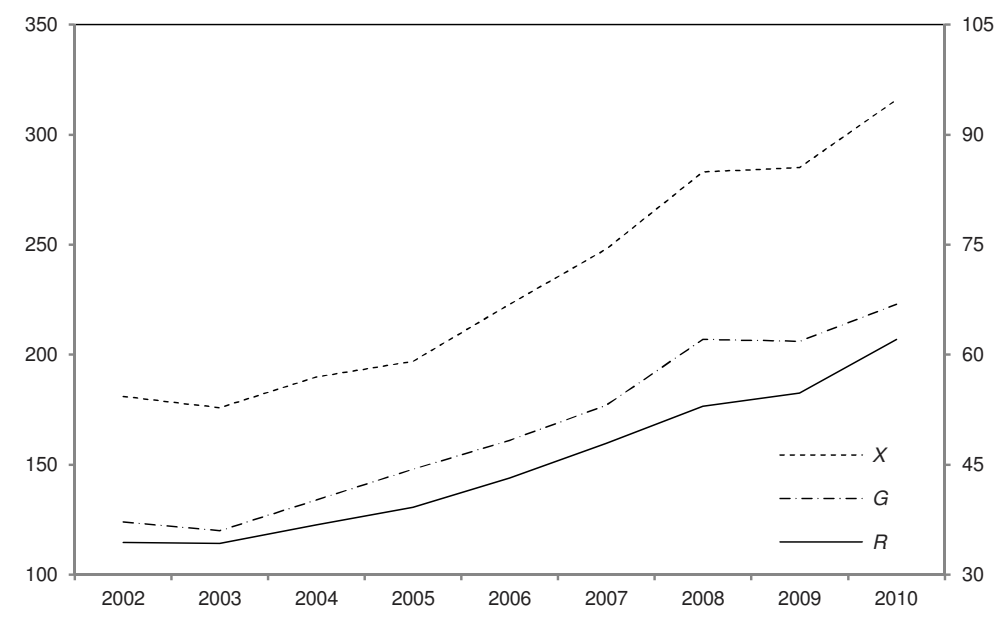

Fonte: FINBRA e RREO's.

Elaboração própria.

* No eixo da direita ficam referenciados os valores das "receitas tributárias $-R$ ".

Figura 1: Evolução dos agregados de receitas e despesas municipais* ${ }^{*} \mathrm{R} \$$ bilhões, 2002 a 2010

[2] Qual é o tamanho da dinâmica nesses dados? Dito de outro modo, quantos anos de defasagens são necessários para resumir a dinâmica nas equações de despesas totais e de arrecadação própria?

[3] Qual é a natureza das relações intertemporais entre despesas orçamentárias, receita tributária e transferências? A dinâmica orçamentária municipal no Brasil pós-LRF é fiel à visão tax and spend de Friedman (1978) ou à hipótese spend and tax de Barro (1979) e Peacock \& Wiseman (1979)?

[4] Qual é o impacto das receitas de transferências sobre o esforço de arrecadação tributária e os gastos totais? O VAR-painel traz evidências de que as transferências intergovernamentais impactam a arrecadação própria e as despesas dos municípios?

Para implementar essa sequência de testes de hipóteses, adotou-se uma estratégia econométrica bastante difundida na literatura (Holtz-Eakin et al. 1989, Dahlberg \& Johansson 1998). Tal estratégia é formada pelos seguintes passos: i) definir um número suficientemente grande de defasagens; ii) estimar o modelo irrestrito, em que nenhum parâmetro é tempo-invariante (equação 4); iii) estimar o modelo restrito, de parâmetros fixos (equação 2), e testar se os coeficientes são estáveis; iv) partindo do modelo selecionado, testar se o número de defasagens pode ser reduzido; $v$ ) aplicar os testes de exclusão de Granger.

É importante ter claro que a mesma matriz de covariância foi utilizada em todas as estimações, para se computar a SSR e aplicar os testes de hipóteses. Essa matriz é a estimativa consistente do segundo estágio da estimação 3SLS proposta por Holtz-Eakin et al. (1988), pressupondo estacionariedade dos parâmetros e que a dinâmica nos dados pode ser resumida em no máximo 4 anos (linha ii nas Tabelas 3 e 4). Um ponto adicional levantado por HoltzEakin et al. (1989) diz respeito ao nível de significância dos testes. Tendo em 
Tabela 3: Equação das despesas orçamentárias $(N=5.544, T=9)$

\begin{tabular}{lrccrc}
\hline & $Q$ & G.L.Q & $L$ & G.L.L & $\chi^{2 a}$ \\
\hline i. Parâmetros variando $(m=4)$ & 15,46 & 9 & - & - & - \\
ii. Parâmetros fixos & 189,42 & 42 & $173,96^{* * *}$ & 33 & 43,75 \\
iii. $m=3$ (dado $i$ ) & 23,74 & 18 & 8,28 & 9 & 14,68 \\
iv. $m=2$ (dado $i)$ & 62,59 & 27 & $38,85^{* * *}$ & 9 & 14,68 \\
v. Excluir $R($ dado iii) & 58,84 & 30 & $35,10^{* * *}$ & 12 & 21,03 \\
vi. Excluir G (dado iii) & 46,34 & 30 & $22,60^{* *}$ & 12 & 21,03 \\
\hline
\end{tabular}

Elaboração própria.

${ }^{* *}$ Rejeita $H_{0}$ a $5 \%,{ }^{* * *}$ rejeita a $1 \%$.

${ }^{a}$ Os valores críticos da distribuição $\chi^{2}$ na última coluna são referentes a níveis de significância de $10 \%$ para os testes de estacionariedade dos parâmetros e do número de defasagens, e 5\% para os testes de Granger.

vista o objetivo final de se testar Granger-causalidade, não é recomendável reduzir muito o modelo. Isso porque a hipótese nula de não causalidade está aninhada com as hipóteses referentes à estacionariedade dos coeficientes e ao tamanho da dinâmica. Quanto menor o modelo, mais terá de ser explicado pelas variáveis remanescentes do lado direto das equações e, portanto, menor a chance de se rejeitar não causalidade. A regra de bolso sugerida pelos autores é adotar um nível de significância de $10 \%$ ao se testar a estabilidade dos coeficientes e o número de defasagens, e $5 \%$ nos testes de exclusão de Granger. $\mathrm{Na}$ última coluna das Tabelas 3 e 4 aparecem os valores críticos referentes a estes níveis de significância.

Começamos por analisar a equação de despesas orçamentárias. Os resultados aparecem na Tabela 3. Como os testes foram realizados pressupondo uma defasagem máxima de 4 anos, isso significa que, na prática, trabalhamos com os 3 últimos anos do período amostral (ou seja, de 2008 a 2010) para respeitar a condição de ordem. Na regressão de parâmetros variáveis, há 48 parâmetros a serem estimados ( 15 coeficientes de dinâmica para cada um dos 3 anos, além dos interceptos) a partir de um total de 57 variáveis instrumentais (há 7, 6 e 5 valores passados de cada uma das 3 variáveis disponíveis como instrumentos em 2010, 2009 e 2008, respectivamente, além de um vetor de constantes em cada ano), resultando num total de 9 graus de liberdade. Na estimação com parâmetros fixos, o número de parâmetros a serem estimados cai para 15 (sendo 12 coeficientes de dinâmica, 1 constante e 2 dummies de tempo) e o número de graus de liberdade é igual a 42. Isso implica existirem 33 restrições neste modelo restrito. Comparando a $S S R$, tem-se uma estatística $L$ de 173,96 $\left(Q_{R}=189,42\right.$ e $\left.Q=15,46\right)$, o que, para 33 graus de liberdade, implica muito fortemente a rejeição do modelo restrito (dado que o valor crítico a $10 \%$ de significância é 43,75).

Cabe notar que a estatística do teste conjunto de especificação e validade das condições de momento (teste de sobreidentificação) é muito elevada para o modelo de parâmetros tempo-invariantes. O primeiro ponto a ser destacado, portanto, é de que existem fortes evidências de não estacionariedade dos parâmetros das equações de despesas orçamentárias. É importante observar também que essas estimativas cobrem o período da crise do subprime e das eleições municipais, havendo evidências de que o comportamento fiscal das prefeituras brasileiras foi influenciado por estes fatos que marcam o período.

O próximo passo consistiu no teste para checar se é possível reduzir o nú- 
Tabela 4: Equação das receitas tributárias $(N=5.544, T=9)$

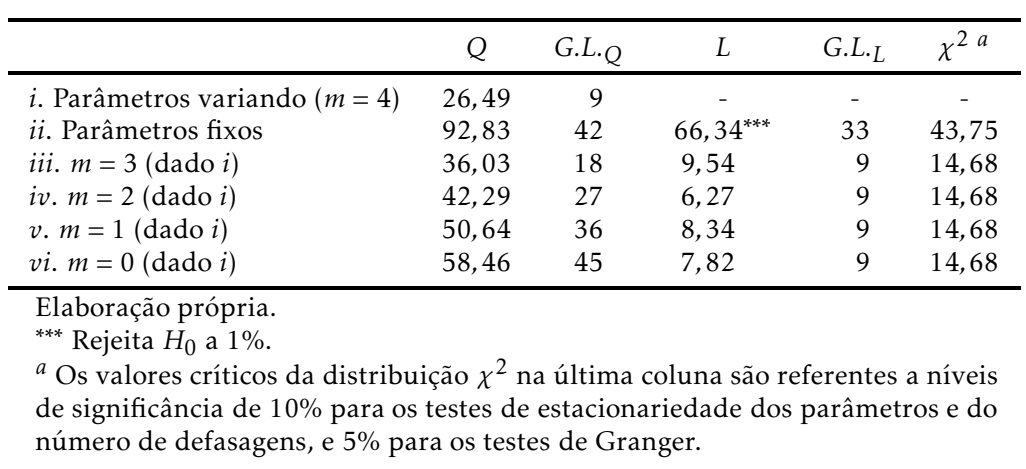

mero de defasagens no modelo, levando em conta a não estabilidade dos parâmetros. As equações com 3 defasagens impõem um total de 9 restrições $(3 \mathrm{em}$ cada ano) sobre o modelo. A estatística $L$ obtida pela comparação dos testes de sobreidentificação não rejeita a hipótese nula de que a quarta defasagem pode ser excluída das equações. O número ótimo de defasagens é aparentemente 3, dado que o acréscimo na $S S R$ é grande quando a estimação foi feita com apenas 2 defasagens.

Os testes de exclusão de Granger foram então aplicados no modelo de parâmetros variáveis com 3 defasagens de cada regressor, incluindo os valores passados da variável dependente. Tais resultados são evidenciados nas últimas duas linhas da Tabela 3.

Ao excluir a arrecadação própria, obteve-se um acréscimo muito significante na $S S R$. A estatística $L$ rejeita a exclusão das receitas tributárias da equação das despesas. Isso significa que o recolhimento de impostos, taxas e contribuições de melhoria, em seu conjunto, precedem no tempo as despesas orçamentárias. Mais adiante examinamos se a recíproca é verdadeira (hipótese de simultaneidade).

Antes disso, checamos se as defasagens das transferências podem ser excluídas do modelo para os gastos totais. Em decorrência do acréscimo produzido na $S S R$ da regressão quando se exclui tal fonte de receitas, pode-se dizer que as transferências precedem as despesas, dado que a estatísticas $L$ é significante. Isso constitui evidência acerca do efeito flypaper porque, mesmo controlando pela arrecadação tributária, as transferências pareceram melhorar a previsão dos gastos totais ${ }^{17,18}$.

Passando para a investigação do modelo das receitas tributárias, começamos por estimar o modelo mais geral, com 4 defasagens e parâmetros variáveis. Novamente, tem-se 48 parâmetros a serem estimados e um total de 57 instrumentos válidos de acordo com as condições de momento ArellanoBond. Como no modelo restrito de parâmetros fixos é necessário estimar apenas 15 coeficientes, tem-se um total de 33 graus de liberdade no teste da hipótese nula de estabilidade paramétrica. A estatística $L$ de 66,34 (dado que $Q_{R}=92,83$ e $\left.Q=26,49\right)$ claramente rejeita tal hipótese.

\footnotetext{
${ }^{17}$ Tal resultado não surpreende, dado que boa parte das transferências encontra-se vinculada, como acontece com os repasses feitos pelo governo federal para as áreas de saúde e educação.

${ }^{18} \mathrm{O}$ apêndice traz os resultados da regressão para as despesas totais, selecionada com base nos testes de exclusão. A equação de receitas tributárias não comparece no apêndice porque os resultados reportados na sequência sugeriram que a arrecadação própria é exógena.
} 
Seguindo o mesmo procedimento adotado no caso do modelo das despesas, o próximo passo foi testar reduções nas defasagens levando em conta a não estacionariedade dos coeficientes. Tais resultados encontram-se na Tabela 4. De uma maneira geral, constatou-se que reduções sucessivas no tamanho da dinâmica desses dados não comprometem a capacidade da regressão predizer as receitas tributárias. A exclusão sucessiva de defasagens nunca produz grandes acréscimos na $S S R$, de tal modo que se atingiu a situação limite de não sobrar dinâmica nos dados. E, por conseguinte, conclui-se que as receitas tributárias são, em média, exógenas com relação às despesas e transferências.

Em síntese, os resultados sugerem as seguintes propriedades para a execução orçamentária municipal: i) há evidências de não estacionariedade na representação encontrada para as despesas; ii) são necessários três anos de dados para resumir as interrelações nas equações dos gastos; iii) tanto a receita própria quanto a de transferências ajudam a prever as despesas totais; e iv) não há dinâmica nos dados de receita tributária, sendo a arrecadação própria aparentemente exógena.

Evidenciam-se, portanto, fatos econômicos importantes acerca da dinâmica orçamentária dos municípios no Brasil. Os gastos públicos dos governos locais acomodam-se, para cima ou para baixo, ao tamanho da arrecadação tributária. E, além disso, mais transferências significam, na prática, novas despesas. $\mathrm{O}$ fato das receitas tributárias parecerem exógenas indica não haver relação evidente entre os gastos em um dado ano e o esforço de recolhimento de impostos nos anos subsequentes. Desta forma, conclui-se que o ajuste no orçamento fica a cargo das despesas, e que novas receitas na forma de arrecadação própria ou transferências intergovernamentais impactam os gastos.

É possível dizer que a visão tax and spend de Friedman (1978) encontra respaldo no comportamento fiscal das prefeituras brasileiras. O mesmo resultado foi encontrado por Holtz-Eakin et al. (1989) nas análises com dados municipais americanos e Dahlberg \& Johansson (2000) que trabalharam com municípios suecos. O resultado de não estacionariedade na equação de despesas foi também encontrado em Dahlberg \& Johansson (1998) e Moisio (2000), bem como em Holtz-Eakin et al. (1989). Finalmente, nas aplicações de Dahlberg \& Johansson (2000), nenhuma defasagem sobreviveu na equação das receitas tributárias, o que mostra que o caso dos municípios brasileiros encontra paralelo em governos locais de outros países. A influência das transferências sobre as despesas, por seu turno, está bem documentada tanto na literatura nacional quanto na internacional.

\section{Considerações finais}

Este trabalho analisou empiricamente as interrelações entre despesas totais, receita própria e transferências correntes por meio de testes de hipóteses aplicados num modelo que combina a estrutura VAR com a ideia do painel de controlar pela heterogeneidade entre as unidades de tomada de decisão.

Os resultados encontrados lançam luz sobre pontos importantes da dinâmica na execução orçamentária municipal. Primeiro, rejeitou-se a hipótese de estacionariedade dos coeficientes nos modelos das despesas totais e receitas tributárias. Segundo, enquanto três anos de defasagens se mostraram necessários para resumir a dinâmica das despesas, nenhuma defasagem sobreviveu aos testes de exclusão aplicados nas regressões para a receita tributária. Ter- 
ceiro, constatou-se que tanto as receitas tributárias como a de transferências melhoram a previsão dos gastos totais.

Tais resultados sugerem que o comportamento fiscal dos municípios brasileiros mudou ao longo do tempo, provavelmente devido a choques macroeconômicos. Em média, existe muito mais dinâmica nos gastos do que na arrecadação própria. E os dados se adéquam à hipótese tax and spend e trazem novas evidências acerca do efeito flypaper. Ou seja, os gastos públicos se acomodam para cima ou para baixo ao tamanho da carga tributária municipal, que é exógena, e as transferências exercem influência própria sobre as despesas.

\section{Agradecimentos}

Este estudo se beneficiou dos códigos de Gauss escritos por Douglas HoltzEakin, bem como da imputação feita por Wesley Silva na base de dados. Registram-se também sinceros agradecimentos a dois árbitros da revista, pelas contribuições. Erros e omissões são de responsabilidade única do autor.

\section{Referências Bibliográficas}

Andrews, D. \& Lu, B. (2001), 'Consistent model and moment selection procedures for GMM estimation with application to dynamic panel data models', Journal of Econometrics 101, 123-164.

Arellano, M. \& Bond, S. (1991), 'Some tests of specification for panel data: Monte Carlo evidence and an application to employment equations', The Review of Economic Studies 58, 277-297.

Arellano, M. \& Bover, O. (1995), 'Another look at the instrumental variable estimation of error-component models', Journal of Econometrics 68, 29-51.

Barro, R. (1979), 'On the determination of public debt', Journal of Political Economy 87(5), 940-971.

Black, D. (1948), 'On the rationale of group decision-making', Journal of Political Economy 56(1), 23-34.

Blundell, R. \& Bond, S. (1998), 'Initial conditions and moment restrictions in dynamic panel data models', Journal of Econometrics 87, 115-143.

Chamberlain, G. (1992), 'Comment: Sequential moment restrictions in panel data', Journal of Business E Economic Statistics 10(1), 20-26.

Chieza, R., Araújo, J. \& Junior, G. S. (2009), 'Impactos da Lei de Responsabilidade Fiscal sobre os componentes da despesa dos municípios gaúchos: análise econométrica', Ensaios FEE 30, 363-390.

Dahlberg, M. \& Johansson, E. (1998), 'The revenues-expenditure nexus: panel data evidence from Swedish municipalities', Applied Economics 30, 13791386.

Dahlberg, M. \& Johansson, E. (2000), 'An examination of the dynamic behavior of local governments using GMM bootstrapping methods', Journal of Applied Econometrics 15, 401-416. 
Dahlberg, M. \& Lindstrom, T. (1998), 'Are local governments governed by forward looking decision makers?', Journal of Urban Economics 44, 254-271.

De Wachter, S. \& Tzavalis, E. (2004), 'Detection of structural breaks in linear dynamics panel data models', London: Queen Mary, University of London. 23 p. Working paper n. 505.

De Wachter, S. \& Tzavalis, E. (2005), 'Monte Carlo comparison of model and moment selection and classical approaches to break detection in panel data models', Economic Letters 88, 91-96.

Friedman, M. (1978), 'The limitations of tax limitation', Policy Review 5, 714.

Holtz-Eakin, D., Newey, W. \& Rosen, H. (1988), 'Estimating vector autoregressions with panel data', Econometrica 56(6), 1371-1395.

Holtz-Eakin, D., Newey, W. \& Rosen, H. (1989), 'The revenues-expenditure nexus: evidence from local government data', International Economic Review 30(3), 415-429.

Hoover, K. \& Sheffrin, S. (1992), 'Causation, spending, and taxes: sand in the sandbox or tax collector for the welfare state?', The American Economic Review 82(1), 225-248.

Mattos, E., Rocha, F. \& Arvate, P. (2011), 'Flypaper effect revisited: evidence for tax collection efficiency in Brazilian municipalities', Estudos Econômicos 41(2), 239-267.

Menezes, R. (2005), 'Impactos da Lei de Responsabilidade Fiscal sobre os componentes da despesa dos municípios brasileiros', Brasília: ESAF. 64 p. Monografia premiada em $1^{\circ}$ lugar no X Prêmio Tesouro Nacional - 2005, Lei de Responsabilidade Fiscal.

Menezes, R. \& Toneto Jr., R. (2006), 'Regras fiscais no Brasil: a influência da LRF sobre as categorias de despesa dos municípios', Planejamento e Políticas Públicas (29), 7-37.

Moisio, A. (2000), 'Spend and tax or tax and spend? panel data evidence from Finnish municipalities during 1985-1999', Helsinki: VATT. 24 p. Working paper n. 242.

Niskanen, W. (1971), Bureaucracy and representative government, AldineAtherton, Chicago.

Orair, R. \& Alencar, A. (2010), 'Esforço fiscal dos municípios: indicadores de condicionalidade para o sistema de transferências intergovernamentais', Brasília: ESAF. 60 p. Monografia premiada em $1^{\circ}$ lugar no XV Prêmio Tesouro Nacional - 2010, Tópicos especiais de finanças públicas.

Orair, R., Santos, C., Silva, W., Brito, M., Silva, H., Rocha, W. \& Ferreira, A. (2011), 'Uma metodologia de construção de séries de alta frequência das finanças municipais no Brasil com aplicação para o IPTU e o ISS: 2004-2010', Pesquisa e Planejamento Econômico 41(3), 471-507. 
Peacock, A. \& Wiseman, J. (1979), 'Approaches to the analysis of government expenditure growth', Public Finance Quarterly 7(1), 3-23.

Postali, F. \& Rocha, F. (2009), 'Resource windfalls, fiscal effort and public spending: evidence from Brazilian municipalities', XXXVII Encontro Nacional de Economia, Foz do Iguaçu, Paraná. 16 p. Trabalho apresentado na reunião anual da Associação Nacional dos Centros de Pós-Graduação em Economia, 2009.

Queiroz, C. \& Postali, F. (2010), 'Rendas do petróleo e eficiência tributária dos municípios brasileiros', XXXVIII Encontro Nacional de Economia, Salvador, Bahia. 18 p. Trabalho apresentado na reunião anual da Associação Nacional dos Centros de Pós-Graduação em Economia, 2010.

Reis, E., Pimentel, M. \& Alvarenga, A. (2007), 'Áreas mínimas comparáveis para os períodos intercensitários de 1872 a 2000', Rio de Janeiro: IPEA.

Roodman, D. (2006), 'How to do xtabond2: an introduction to "difference"and "system"GMM in stata', Washington, DC: Center for Global Development. 42 p. Working paper n. 103.

Santolin, R., Jayme Jr., F. \& Reis, J. (2009), 'Lei de Responsabilidade Fiscal e implicações na despesa de pessoal e de investimento nos municípios mineiros: um estudo com dados em painel dinâmico', Estudos Econômicos 39(4), 895-923.

Wooldridge, J. (1991), 'Multiplicative panel data models without the strict exogeneity assumption', Cambridge, MA: MIT. 23 p. Working paper n. 574. 


\section{Apêndice $A$}

Tabela A.1: Equação para as despesas orçamentárias - forma reduzida, equação $(4)^{a}$

\begin{tabular}{|c|c|c|c|}
\hline & RHS var. & Est. & T-ratio ${ }^{b}$ \\
\hline \multirow[t]{12}{*}{2008} & $X_{t-1}$ & $-0,24$ & 0,64 \\
\hline & $R_{t-1}$ & 1,30 & 0,61 \\
\hline & $G_{t-1}$ & $1,27^{* * *}$ & 3,32 \\
\hline & $X_{t-2}$ & $0,60^{* * *}$ & 3,48 \\
\hline & $R_{t-2}$ & 0,13 & 0,10 \\
\hline & $G_{t-2}$ & $-0,60^{* * *}$ & $-2,84$ \\
\hline & $X_{t-3}$ & 0,05 & 0,49 \\
\hline & $R_{t-3}$ & $-0,61$ & $-1,40$ \\
\hline & $G_{t-3}$ & $-0,09$ & $-0,69$ \\
\hline & $X_{t-4}$ & $0,23^{* * *}$ & 2,67 \\
\hline & $R_{t-4}$ & $-0,03$ & $-0,04$ \\
\hline & $G_{t-4}$ & $-0,20^{* *}$ & $-2,15$ \\
\hline \multirow[t]{12}{*}{2009} & $X_{t-1}$ & $0,79^{* *}$ & 2,53 \\
\hline & $R_{t-1}$ & 2,57 & 0,88 \\
\hline & $G_{t-1}$ & $-0,31$ & $-0,66$ \\
\hline & $X_{t-2}$ & 0,16 & 0,94 \\
\hline & $R_{t-2}$ & $-3,25$ & $-0,92$ \\
\hline & $G_{t-2}$ & 0,41 & 0,84 \\
\hline & $X_{t-3}$ & $-0,07$ & $-0,81$ \\
\hline & $R_{t-3}$ & $0,78^{* *}$ & 2,38 \\
\hline & $G_{t-3}$ & $-0,08$ & $-0,70$ \\
\hline & $X_{t-4}$ & 0,06 & 1,10 \\
\hline & $R_{t-4}$ & $-0,15$ & $-0,41$ \\
\hline & $G_{t-4}$ & $-0,01$ & $-0,11$ \\
\hline \multirow[t]{12}{*}{2010} & $X_{t-1}$ & $1,11^{* * *}$ & 3,06 \\
\hline & $R_{t-1}$ & $-3,19^{* *}$ & $-2,53$ \\
\hline & $G_{t-1}$ & $-0,24$ & $-0,78$ \\
\hline & $X_{t-2}$ & $-0,07$ & $-0,47$ \\
\hline & $R_{t-2}$ & $2,74^{* *}$ & 2,50 \\
\hline & $G_{t-2}$ & 0,34 & 1,49 \\
\hline & $X_{t-3}$ & $-0,01$ & $-0,04$ \\
\hline & $R_{t-3}$ & $-0,56$ & $-1,32$ \\
\hline & $G_{t-3}$ & $-0,05$ & $-0,45$ \\
\hline & $X_{t-4}$ & 0,03 & 0,53 \\
\hline & $R_{t-4}$ & $1,23^{* * *}$ & 3,83 \\
\hline & $G_{t-4}$ & $-0,03$ & $-0,39$ \\
\hline
\end{tabular}

Dep. Var: $X_{t}, \quad N=5.544, T=9, \quad N \times T=49.896$.

Método: GMM, RHS var.: 39, IV's: 57.

Chi-Square Stat. $-Q=23,74, \quad$ G.L. $=18$,

$\operatorname{Prob}(\chi \geq Q)=0,16$.

Elaboração própria. ${ }^{* *}$ Rejeita $H_{0}$ a $5 \%,{ }^{* * *}$ rejeita a $1 \% . \quad{ }^{a}$ Foram omitidas as dummies de tempo. ${ }^{b}$ Erros-padrão com correção de heterocedasticidade - HC. 